\title{
FTIR Spectroscopy and DFT Calculations to Probe the Kinetics of $\beta$-Carotene Thermal Degradation
}

\author{
Daniel Martin,${ }^{\dagger}$ Ana M. Amado,$^{\dagger}$ Alicia G. Gonzálvez, ${ }^{\dagger}$ M. Paula M. Marques, ${ }^{\dagger \#}$ \\ Luís A. E. Batista de Carvalho, ${ }^{\dagger *}$ and Ángel González Ureña \\ †nidade de I\&D Química-Física Molecular, Department of Chemistry, University of Coimbra, 3004-535 \\ Coimbra, PORTUGAL \\ Departamento de Química-Física Aplicada, Facultad de Ciencias, Universidad Autónoma de Madrid, Madrid, \\ SPAIN \\ \#Department of Life Sciences, University of Coimbra, 3000-456 Coimbra, PORTUGAL \\ * Corresponding Author: labc@ci.uc.pt
}

\section{SUPPORTING INFORMATION}

\section{Table of Contents}

Figure S1 - Structural representation of the key intermediates of $\beta$-carotene thermal degradation.

Table S1 - Experimental and calculated infrared wavenumbers and assignment for $\beta$ carotene.

Table S2 - Experimental and calculated infrared wavenumbers and assignment for retinal.

Table S3 - Calculated geometrical parameters for the 9-cis intermediate of $\beta$-carotene thermal degradation.

Table S4 - Calculated geometrical parameters for the 13-cis intermediate of $\beta$-carotene thermal degradation.

Table S5 - Calculated geometrical parameters for the 15-cis intermediate of $\beta$-carotene thermal degradation.

References 

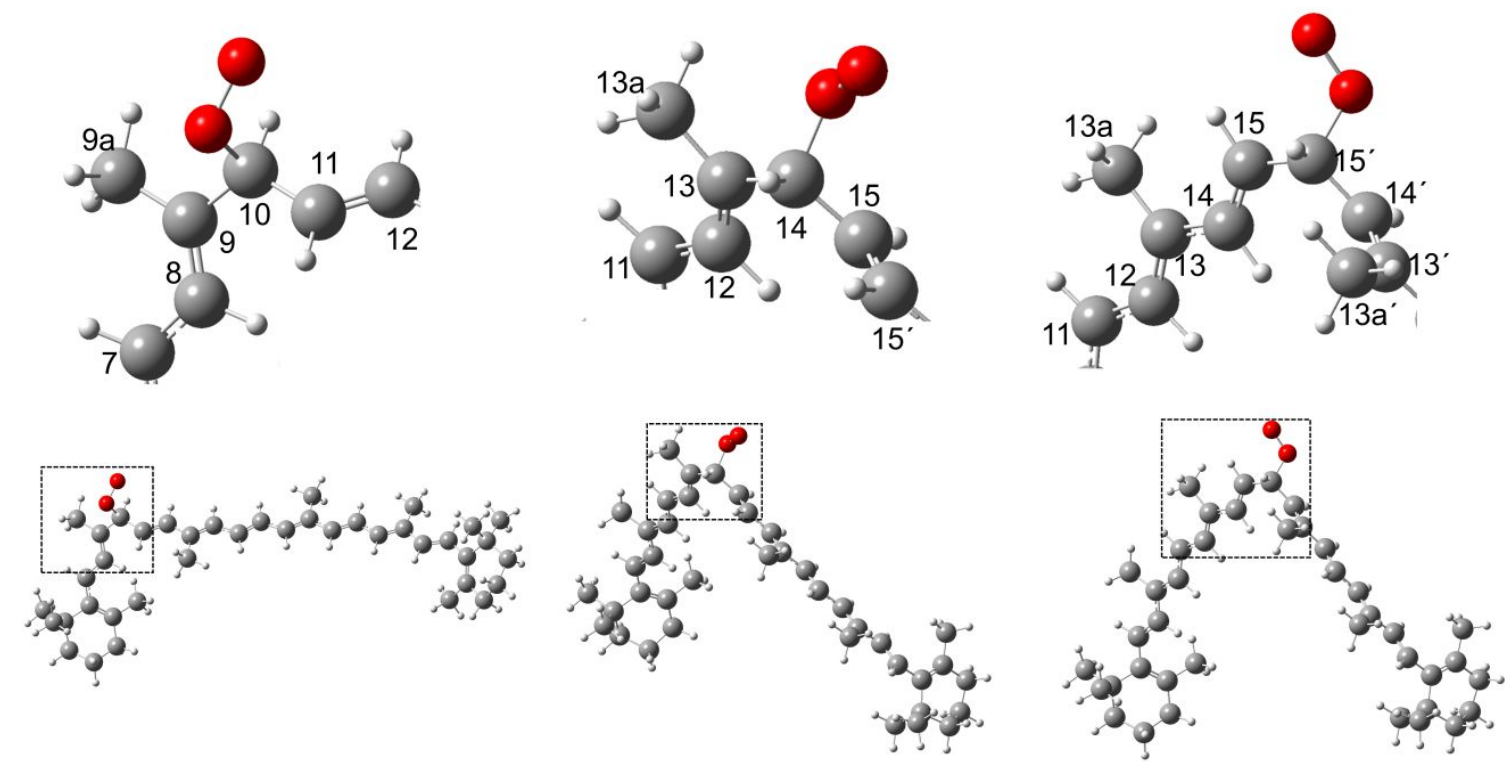

$\underline{\text { Figure } \mathrm{S} 1}$ - Structural representation of the key intermediates of $\beta$-carotene thermal degradation. 
Table S1 - Experimental and calculated infrared wavenumbers $\left(\mathrm{cm}^{-1}\right)$ and assignment for $\beta$-carotene.

\begin{tabular}{cccc}
\hline $\begin{array}{c}\text { Calculated } \\
\text { (uncorrected })\end{array}$ & $\begin{array}{c}a_{\text {Calculated }} \\
\text { (corrected })\end{array}$ & Experimental & Assignment \\
\hline 1017 & 1000 & 950 & $\omega_{\mathrm{OP}}(\mathrm{C}-\mathrm{H})$ \\
1038 & 1020 & - & $\delta_{\text {asym }}\left(\mathrm{CH}_{3}\right)$ \\
- & - & 966 & $\omega_{\mathrm{OP}}(\mathrm{C}=\mathrm{C}-\mathrm{H})(\mathrm{C}-\mathrm{C}-\mathrm{Me})^{2}$ \\
1221 & 1200 & 1172 & $v(\mathrm{C}-\mathrm{C})$ \\
$1418 ; 1428$ & $1393 ; 1403$ & 1367 & $\delta_{\text {sym }}\left(\mathrm{CH}_{3}\right)^{3}$ \\
1509 & 1483 & 1455 & $\delta(\mathrm{CH})^{3}$ \\
$1652 ; 1659$ & $1623 ; 1630$ & 1559 & $v(\mathrm{C}=\mathrm{C})$ \\
- & - & 1673 & $v(\mathrm{C}=\mathrm{C}-\mathrm{C})$ \\
1718 & 1.688 & - & $v(\mathrm{C}=\mathrm{C})$ ring \\
3016-3221 & - & 1724 & $v(\mathrm{C}=\mathrm{C}-\mathrm{C})$ \\
\hline${ }^{a}$ ref. $1 . \omega-$ wagging; $v-$ stretching; $\delta-$ deformation; sym- symmetric; asym-antisymmetric; op - out-of-plane.
\end{tabular}

Table S2 - Experimental and calculated infrared wavenumbers $\left(\mathrm{cm}^{-1}\right)$ and assignment for retinal.

\begin{tabular}{cccc}
\hline $\begin{array}{c}\text { Calculated } \\
\left(\mathrm{cm}^{-1}, \text { uncorrected }\right)\end{array}$ & $\begin{array}{c}\text { Calculated } \\
\left(\mathrm{cm}^{-1},{ }^{a} \text { corrected }\right)\end{array}$ & $\begin{array}{c}\text { Experimental } \\
\left(\mathrm{cm}^{-1}, \text { products mixture }\right)\end{array}$ & Assignment \\
\hline 1016 & 995 & 967 & $\omega_{\mathrm{OP}}(\mathrm{C}-\mathrm{H})$ \\
1018 & 1002 & - & $\delta_{\text {asym }}\left(\mathrm{CH}_{3}\right)$ \\
- & - & 975 & $\omega_{\mathrm{OP}}(\mathrm{C}-\mathrm{H})$ \\
1213 & 1191 & 1.235 & $v(\mathrm{C}-\mathrm{C})$ \\
$1384 ; 1419 ; 1449$ & $1364 ; 1396 ; 1427$ & $1343-1402$ & $\delta_{\text {sym }}\left(\mathrm{CH}_{3}\right)^{3}$ \\
1521 & 1497 & $1402-1496$ & $\delta_{\text {asym }}\left(\mathrm{CH}_{3}\right)$ \\
1649 & 1639 & 1629 & $v(\mathrm{C}=\mathrm{C})$ \\
1796 & 1765 & 1713 & $v(\mathrm{C}=\mathrm{O})$ \\
2970 & 2920 & 2870 & $v\left(\mathrm{C}_{\mathrm{C}=\mathrm{O}-\mathrm{H})}\right.$ \\
$3027-3225$ & $2975-3172$ & $2886-3026$ & $v(\mathrm{CH})$ \\
\hline
\end{tabular}

${ }^{a}$ ref. 1. $\omega$ - wagging; $v$ - stretching; $\delta$-deformation; sym-symmetric; asym-antisymmetric; op - out-of-plane. 
Table S3 - Calculated geometrical parameters for the 9-cis intermediate of $\beta$-carotene thermal degradation.

\begin{tabular}{|c|c|c|c|c|c|}
\hline \multicolumn{6}{|c|}{ 9-cis $\beta$-carotene $\mathrm{O}_{2}$ diradical } \\
\hline Distance $(\AA)$ & & Angle $\left(^{\circ}\right)$ & & Dihedral Angle $\left(^{\circ}\right)$ & \\
\hline${ }^{a} \mathrm{C}(7)-\mathrm{C}(8)$ & 1.405 & $\mathrm{C}(7)-\mathrm{C}(8)-\mathrm{C}(9)$ & 125.6 & $\mathrm{C}(7)-\mathrm{C}(8)-\mathrm{C}(9)-\mathrm{C}(9 \mathrm{a})$ & -4.4 \\
\hline $\mathrm{C}(8)-\mathrm{C}(9)$ & 1.378 & $\mathrm{C}(8)-\mathrm{C}(9)-\mathrm{C}(9 \mathrm{a})$ & 123.7 & $\mathrm{C}(9 \mathrm{a})-\mathrm{C}(9)-\mathrm{C}(10)-\mathrm{O}$ & 52.6 \\
\hline $\mathrm{C}(9)-\mathrm{C}(9 \mathrm{a})$ & 1.501 & $C(8)-C(9)-C(10)$ & 121.0 & $C(9 a)-C(9)-C(10)-C(11)$ & 172.7 \\
\hline $\mathrm{C}(9)-\mathrm{C}(10)$ & 1.500 & $C(9 a)-C(9)-C(10)$ & 115.2 & $C(7)-C(8)-C(9)-C(10)$ & 176.5 \\
\hline $\mathrm{C}(10)-\mathrm{O}$ & 1.501 & $\mathrm{C}(9)-\mathrm{C}(10)-\mathrm{O}$ & 105.1 & $\mathrm{C}(8)-\mathrm{C}(9)-\mathrm{C}(10)-\mathrm{O}$ & -128.2 \\
\hline $\mathrm{O}-\mathrm{O}$ & 1.302 & $\mathrm{C}(10)-\mathrm{O}-\mathrm{O}$ & 111.6 & $\mathrm{C}(9)-\mathrm{C}(10)-\mathrm{O}-\mathrm{O}$ & -152.8 \\
\hline$C(10)-C(11)$ & 1.486 & $\mathrm{C}(9)-\mathrm{C}(10)-\mathrm{C}(11)$ & 117.8 & $\mathrm{O}-\mathrm{O}-\mathrm{C}(10)-\mathrm{C}(11)$ & 80.7 \\
\hline $\mathrm{C}(11)-\mathrm{C}(12)$ & 1.345 & $\mathrm{C}(10)-\mathrm{C}(11)-\mathrm{C}(12)$ & 122.7 & $\mathrm{O}-\mathrm{C}(10)-\mathrm{C}(11)-\mathrm{C}(12)$ & -111.7 \\
\hline
\end{tabular}

Table S4 - Calculated geometrical parameters for the 13-cis intermediate of $\beta$-carotene thermal degradation.

\begin{tabular}{|c|c|c|c|c|c|}
\hline \multicolumn{6}{|c|}{ 13-cis $\beta$-carotene $\mathrm{O}_{2}$ diradical } \\
\hline Distance $(\AA)$ & & Angle $\left(^{\circ}\right)$ & & Dihedral Angle $\left(^{\circ}\right)$ & \\
\hline${ }^{a} \mathrm{C}(11)-\mathrm{C}(12)$ & 1.422 & $\mathrm{C}(11)-\mathrm{C}(12)-\mathrm{C}(13)$ & 127.3 & $C(11)-C(12)-C(13)-C(13 a)$ & 0.1 \\
\hline $\mathrm{C}(12)-\mathrm{C}(13)$ & 1.363 & $\mathrm{C}(12)-\mathrm{C}(13)-\mathrm{C}(13 \mathrm{a})$ & 124.7 & $C(13 a)-C(13)-C(14)-O$ & -51.6 \\
\hline$C(13)-C(13 a)$ & 1.500 & $C(12)-C(13)-C(14)$ & 120.6 & $C(13 a)-C(13)-C(14)-C(15)$ & -172.0 \\
\hline $\mathrm{C}(13)-\mathrm{C}(14)$ & 1.504 & $C(13 a)-C(13)-C(14)$ & 114.7 & $\mathrm{C}(11)-\mathrm{C}(12)-\mathrm{C}(13)-\mathrm{C}(14)$ & 178.0 \\
\hline $\mathrm{C}(14)-\mathrm{O}$ & 1.496 & $C(13)-C(14)-O$ & 105.5 & $\mathrm{C}(12)-\mathrm{C}(13)-\mathrm{C}(14)-\mathrm{O}$ & 130.3 \\
\hline $\mathrm{O}-\mathrm{O}$ & 1.303 & $\mathrm{C}(14)-\mathrm{O}-\mathrm{O}$ & 111.6 & $\mathrm{C}(13)-\mathrm{C}(14)-\mathrm{O}-\mathrm{O}$ & 151.5 \\
\hline$C(14)-C(15)$ & 1.486 & $C(13)-C(14)-C(15)$ & 117.6 & $\mathrm{O}-\mathrm{O}-\mathrm{C}(14)-\mathrm{C}(15)$ & -82.0 \\
\hline$C(15)-C\left(15^{\prime}\right)$ & 1.346 & $C(14)-C(15)-C\left(15^{\prime}\right)$ & 123.2 & $\mathrm{O}-\mathrm{C}(14)-\mathrm{C}(15)-\mathrm{C}\left(15^{\prime}\right)$ & 113.7 \\
\hline
\end{tabular}

Table S5 - Calculated geometrical parameters for the 15-cis intermediate of $\beta$-carotene thermal degradation.

\begin{tabular}{|c|c|c|c|c|c|}
\hline \multicolumn{6}{|c|}{15 -cis $\beta$-carotene $\mathrm{O}_{2}$ diradical } \\
\hline Distance/ $\AA$ & & Angle $/^{\circ}$ & & Dihedral Angle/ ${ }^{\circ}$ & \\
\hline${ }^{a} \mathrm{C}(11)-\mathrm{C}(12)$ & 1.405 & $\mathrm{C}(11)-\mathrm{C}(12)-\mathrm{C}(13)$ & 128.2 & $\mathrm{C}(11)-\mathrm{C}(12)-\mathrm{C}(13)-\mathrm{C}(13 \mathrm{a})$ & -0.2 \\
\hline $\mathrm{C}(12)-\mathrm{C}(13)$ & 1.384 & C(12)-C(13)-C(13a) & 123.4 & $C(13 a)-C(13)-C(14)-C(15)$ & 0.7 \\
\hline $\mathrm{C}(13)-\mathrm{C}(13 \mathrm{a})$ & 1.500 & $C(12)-C(13)-C(14)$ & 118.0 & $\mathrm{C}(12)-\mathrm{C}(13)-\mathrm{C}(14)-\mathrm{C}(15)$ & -179.3 \\
\hline $\mathrm{C}(13)-\mathrm{C}(14)$ & 1.441 & C(13a)-C(13)-C(14) & 118.6 & $\mathrm{C}(11)-\mathrm{C}(12)-\mathrm{C}(13)-\mathrm{C}(14)$ & 179.9 \\
\hline $\mathrm{C}(14)-\mathrm{C}(15)$ & 1.350 & $\mathrm{C}(13)-\mathrm{C}(14)-\mathrm{C}(15)$ & 125.9 & $\mathrm{C}(13)-\mathrm{C}(14)-\mathrm{C}(15)-\mathrm{C}\left(15^{\prime}\right)$ & 178.3 \\
\hline$C(15)-C\left(15^{\prime}\right)$ & 1.497 & $\mathrm{C}(14)-\mathrm{C}(15)-\mathrm{C}\left(15^{\prime}\right)$ & 124.5 & $\mathrm{C}(14)-\mathrm{C}(15)-\mathrm{C}\left(15^{\prime}\right)-\mathrm{O}$ & 133.9 \\
\hline $\mathrm{C}\left(15^{\prime}\right)-\mathrm{O}$ & 1.490 & $\mathrm{C}(15)-\mathrm{C}\left(15^{\prime}\right)-\mathrm{O}$ & 108.1 & $\mathrm{C}(14)-\mathrm{C}(15)-\mathrm{C}\left(15^{\prime}\right)-\mathrm{C}\left(14^{\prime}\right)$ & 16.4 \\
\hline $\mathrm{O}-\mathrm{O}$ & 1.303 & $\mathrm{C}\left(15^{\prime}\right)-\mathrm{O}-\mathrm{O}$ & 111.2 & $\mathrm{C}(15)-\mathrm{C}\left(15^{\prime}\right)-\mathrm{O}-\mathrm{O}$ & 67.8 \\
\hline $\mathrm{C}\left(15^{\prime}\right)-\mathrm{C}\left(14^{\prime}\right)$ & 1.488 & $\mathrm{O}-\mathrm{C}\left(15^{\prime}\right)-\mathrm{C}\left(14^{\prime}\right)$ & 105.0 & $\mathrm{O}-\mathrm{O}-\mathrm{C}\left(15^{\prime}\right)-\mathrm{C}\left(14^{\prime}\right)$ & -168.0 \\
\hline $\mathrm{C}\left(14^{\prime}\right)-\mathrm{C}\left(13^{\prime}\right)$ & 1.351 & $\mathrm{C}\left(15^{\prime}\right)-\mathrm{C}\left(14^{\prime}\right)-\mathrm{C}\left(13^{\prime}\right)$ & 127.7 & $\mathrm{O}-\mathrm{C}\left(15^{\prime}\right)-\mathrm{C}\left(14^{\prime}\right)-\mathrm{C}\left(13^{\prime}\right)$ & 126.8 \\
\hline$C\left(13^{\prime}\right)-C\left(13 a^{\prime}\right)$ & 1.504 & $\mathrm{C}\left(14^{\prime}\right)-\mathrm{C}\left(13^{\prime}\right)-\mathrm{C}\left(13 \mathrm{a}^{\prime}\right)$ & 124.0 & $\mathrm{C}\left(15^{\prime}\right)-\mathrm{C}\left(14^{\prime}\right)-\mathrm{C}\left(13^{\prime}\right)-\mathrm{C}\left(13 \mathrm{a}^{\prime}\right)$ & 0.8 \\
\hline
\end{tabular}




\section{REFERENCES}

1. Merrick, J. P.; Moran, D.; Radom, L. An Evaluation of Harmonic Vibrational Frequency Scale Factors. J. Phys. Chem. A., 2007, 111, 11683-11700.

2. Schlücker, S.; Szeghalmi, A.; Schmitt, M.; Popp, J.; Kiefer, W. Dencsity Functional and Vibrational Spectroscopic Analysis of $\beta$-Carotene. J. Raman Spectrosc., 2003, 34 (6), 413-419.

3. Baranska, M.; Schütze, W.; Schulz, H. Determination of Lycopene and $\beta$-Carotene Content in Tomato Fruits and Related Products: Comparison of FT-Raman, ATR-IR, and NIR Spectroscopy. Anal Chem., 2006, 78(24), 8456-8461.

4. Finkelstein, E. I.; Krasnokutskaya, I. S.; Vakulova, L. A. Spectroscopy of Carotenoids and its Application to the Investigation of Autoxidation. J. Mol. Struct., 1999, 480-481, 569-571. 